\title{
SPECT Low-Field MRI System for Small-Animal Imaging
}

\author{
Christian Goetz ${ }^{1}$, Elodie Breton ${ }^{1}$, Philippe Choquet $^{1}$, Vincent Israel-Jost ${ }^{2}$, and André Constantinesco ${ }^{1}$ \\ ${ }^{1}$ Service de Biophysique et Médecine Nucléaire, Hôpital de Hautepierre, Strasbourg, France; and ${ }^{2}$ ENST GET/TSI, Paris, France
}

\begin{abstract}
Localization of regions with increased uptake of radiotracer in small-animal SPECT is greatly facilitated when using coregistration with anatomic images of the same animal. As MRI has several advantages compared with CT (soft-tissue contrast and lack of ionizing radiation) we developed a SPECT/low-field MRI hybrid device for small-animal imaging. Methods: A small-animal single-pinhole $\gamma$-camera (pinhole, $1.5 \mathrm{~mm}$ in diameter and $12 \mathrm{~cm}$ in focal length) adjacent to a dedicated low-field $(0.1 \mathrm{~T})$ small MR imager (imaging volume, $10 \times 10 \times 6 \mathrm{~cm}^{3}$ ) was used. The animal was placed in a warmed nonmagnetic polymethyl methacrylate imaging cell for MR acquisition, which was followed immediately by SPECT after translation of the imaging cell from one modality to the other. 3-Dimensional T1-weighted sequences were used for MRI. Phantom studies enabled verifica-

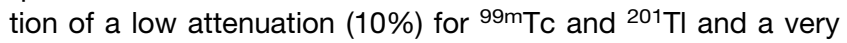
slight increase in Compton scattering due to the radiofrequency coil and polymethyl methacrylate imaging cell. Results: SPECT/ MRI data acquisition and image coregistration of selected examples using different radiotracers for lungs, kidneys, and brain were obtained in 3 nude mice with isotropic spatial resolutions of $0.5 \times 0.5 \times 0.5 \mathrm{~mm}^{3}$ for MRI and $1 \times 1 \times 1 \mathrm{~mm}^{3}$ for SPECT. The total acquisition time for combined SPECT and MRI lasted $1 \mathrm{~h} 45$ min. Conclusion: A low-magnetic-field strength of $0.1 \mathrm{~T}$ is a simple and useful solution for a small-animal dual-imaging device combining pinhole SPECT with the adjacent MR imager.
\end{abstract}

Key Words: small animals; dual modality; SPECT; MRI; molecular imaging

J Nucl Med 2008; 49:88-93

DOI: 10.2967/jnumed.107.044313

\section{A}

mong small-animal imaging techniques, SPECT provides a unique possibility to follow and measure in vivo, and noninvasively, the biodistribution of a $10^{-9}$ molar concentration of a wide range of radiolabeled biomolecules commonly available in nuclear medicine departments (1). However, one essential drawback in SPECT images is the lack of anatomic references related to the tissue uptake of tracer. Therefore, localization of regions with increased uptake of radiotracer is greatly facilitated when using

\footnotetext{
Received Jun. 19, 2007; revision accepted Oct. 12, 2007.

For correspondence or reprints contact: André Constantinesco, MD, PhD, Service de Biophysique et Médecine Nucléaire, CHU Hautepierre, 1 av. Molière, 67098, Strasbourg, France.

E-mail: andre.constantinesco@chru-strasbourg.fr

COPYRIGHT $\odot 2008$ by the Society of Nuclear Medicine, Inc.
}

coregistration of SPECT with anatomic images of the same animal, from CT or MRI. Over the last few years, SPECT/ CT dual modality has been widely proposed by manufacturers but MRI presents specific advantages compared with CT, including lack of ionizing radiation, high soft-tissue contrast, and sensitivity to tissue alterations evidenced by specific imaging sequences $(2,3)$. Yet, compared with CT, the use of MRI for coregistration of both functional and structural information has, to our knowledge, essentially served to demonstrate the potential interest of using coregistration of images after data acquisition in separate nuclear and MR rooms. However, as experienced for rat and mice, the strategy of pinhole SPECT followed by MRI in a clinical scanner is a long and complicated task (4-7), requiring a careful transfer of the animal in a specially designed bed equipped with multimodality fiducial markers helping in the coregistration of images $(4,5)$. In addition, separate dual-modality scans (followed by image fusion) are also potential sources of misalignment of images due to uncontrolled movements and displacements of tissues and organs. Moreover, multiple anesthesia sessions are needed when SPECT and MRI devices are located in separate places, involving the risk of different biologic responses to anesthesia (3). After early rodent MRI works done on clinical low-field systems operating at $0.35 \mathrm{~T}(8)$, smallanimal MRI is now performed exclusively at very high magnetic fields operating at values up to $14 \mathrm{~T}$, in a desire to achieve high anatomic resolution benefiting from a higher signal-to-noise ratio but leading to field susceptibility artifacts and preventing close proximity of the SPECT scanner $(2,9)$. As for PET/MRI, technical limitations for dual SPECT/MRI systems are therefore primarily due to complex physical interactions between the high magnetic field of the MR device and nuclear instrumentation. Two strategies are then possible for the design of a SPECT/MRI dual-modality system for small-animal imaging. The first strategy is the development of high-magnetic-field MR-compatible SPECT inserts as was recently proposed at $7 \mathrm{~T}(10)$. However, a similar approach for MR-compatible PET inserts resulted in the costly design of small-animal prototypes for simultaneous dual-modality devices working for MRI at 4.7 and $7 \mathrm{~T}$ $(11,12)$. The second strategy is the use of a low magnetic field for MRI that could have the combined advantages of avoiding field strength imaging artifacts and interactions 
with the SPECT instrumentation while providing sufficient pixel resolution for an appropriate routine solution for a small-animal dual-imaging device that would minimize time and distance between the scans. After a previous proof-of-concept study (13), in this article we propose to describe, illustrate, and discuss our practical experience in small-animal sequential SPECT/MR image acquisition and fusion using a coaxially back-to-back configuration of a pinhole SPECT with a 0.1-T MRI device.

\section{MATERIALS AND METHODS}

\section{Animal Holder and Anesthesia}

All animal experiments were conducted in compliance with the French guidelines for the care and use of research animals (authorization 67-482-20). Mice were maintained under gaseous anesthesia $\left(1 \%-1.5 \%\right.$ isoflurane in air) in an air-warmed $\left(40^{\circ} \mathrm{C}\right)$, nonmagnetic technical cell dedicated to small-animal imaging and adapted for both SPECT and MRI devices (CTI; Minerve) (Fig. 1). The imaging cell isolates the animal during image acquisition of both modalities. The use of the imaging cell equipped with a single radiofrequency (RF) transmit-and-receive coil allows an easy and immediate transfer of the cell from one imaging system to the other while keeping the animal in the same position. Therefore, fiducial markers in the imaging cell were avoided in our experiments.

\section{SPECT System and Image Reconstruction}

For SPECT we used a small-animal dedicated rotating singlehead $\gamma$-camera with a 1.5 -mm-diameter tungsten pinhole collimator and $12 \mathrm{~cm}$ in focal distance (Gaede). For small-animal SPECT image acquisition, a $20 \%$ window centered on the $140-\mathrm{keV}$ photopeak was used, and 60 projections of 1 min over an arch of $180^{\circ}$ in a $128 \times 128$ format were obtained using a $4-\mathrm{cm}$ radius of rotation around the imaging cell. Images were reconstructed with an inhouse efficient algebraic cone beam reconstruction method based on an algebraic reconstruction technique (ART) algorithm that takes into account the variation of the radius of rotation and is able to achieve an isotropic $1 \times 1 \times 1 \mathrm{~mm}^{3}$ spatial resolution (efficient algebraic reconstruction [EAR] method (14)). The size of the isotropic reconstructed voxel was $0.47 \times 0.47 \times 0.47 \mathrm{~mm}^{3}$. The cost of the complete pinhole SPECT apparatus was around $\$ 50,000$.

\section{MRI System and Design of RF Coils}

MRI was performed with a small water-cooled low-field (main magnetic field strength $\mathrm{B}_{0}=0.1 \mathrm{~T}$ oriented vertically) open electromagnet (Bouhnik SAS) that was previously described in detail (15). It has a homogeneous (relative variation of magnetic field strength $\Delta \mathrm{B} / \mathrm{B}_{0}=5.10^{-6}$ ) imaging volume of $10 \times 10 \times 6$ $\mathrm{cm}^{3}$ and maximum encoding gradients' strength of $20 \mathrm{mT} / \mathrm{m}$ that can be reached in $<0.5 \mathrm{~ms}$. RF transmit-and-receive solenoidal coils (for whole-body or brain images) were made by adjacent loops of a 0.7 -mm-diameter copper wire rounded on a thin $(0.2$ $\mathrm{mm})$ plastic cylinder to minimize attenuation and scattering of $\gamma$-rays during SPECT. The animal was placed from the beginning in the imaging cell as illustrated in Figure 1 and kept in the same position during both acquisitions. RF pulses are transmitted to the solenoidal coil by a pickup copper coil rounded outside the imaging cell (as illustrated in Fig. 1), which also received the encoded MR signal. A T1-weighted 3-dimensional (3D) sequence was developed on a SMIS (Guilford, U.K.) console to achieve isotropic spatial resolution of $0.5 \times 0.5 \times 0.5 \mathrm{~mm}^{3}$. T1-weighted isotropic acquisitions were obtained in $42 \mathrm{~min}$ with echo time $(\mathrm{TE})=7 \mathrm{~ms}$, repetition time $(\mathrm{TR})=23 \mathrm{~ms}$, flip angle $(\mathrm{FA})=60^{\circ}$, $128 \times 64 \times 48$ or $256 \times 64 \times 48$ matrix, number of excitations $(\mathrm{NEX})=36$, and field of view $(\mathrm{FOV})=64 \times 32 \times 32 \mathrm{~mm}^{3}$ or $128 \times$ $32 \times 32 \mathrm{~mm}^{3}$. The cost of the complete MR equipment was around $\$ 200,000$.

\section{Combined Sequential SPECT/MRI Dual Modality}

As the sizes of our 2 imaging devices (SPECT: 103-cm length by $80-\mathrm{cm}$ width and $140-\mathrm{cm}$ height; low-field MRI: 72-cm length by $72-\mathrm{cm}$ width and $118-\mathrm{cm}$ height) fit in a single room, and as the 5-Gauss line of the magnetic field is located at $15 \mathrm{~cm}$ from the sides of the magnet, the pinhole SPECT camera was placed directly close and coaxially to the MR magnet as well as the anesthesia equipment without any adverse effects between them (as illustrated in Fig. 1). After MR acquisition, the pickup coil was removed, and the imaging cell containing the animal and the RF coil was manually translated, on the common $z$-axis, from the center of the magnet to the pinhole SPECT system and docked in $<1 \mathrm{~min}$.

\section{Phantom Studies}

The influence of the RF coil and the polymethyl methacrylate tube wall of the imaging cell on attenuation and scattering of $\gamma$-rays was tested for 2 different isotopes in SPECT projection images of a simple tube filled with $37 \mathrm{MBq}$ of a solution of ${ }^{99 \mathrm{~m}} \mathrm{Tc}$ and ${ }^{201} \mathrm{Tl}$, respectively. Windows of $20 \%$ centered on $140 \mathrm{keV}$ for the ${ }^{99 \mathrm{~m}} \mathrm{Tc}$ and on 71 and $167 \mathrm{keV}$ for the ${ }^{201} \mathrm{Tl}$, respectively, were used. A second phantom consisting in 3 tubes of different diameters, described in Figure 2A and placed inside the imaging cell, was used to set out the centering errors consecutive to the manual translation of the imaging chamber from one imaging modality to
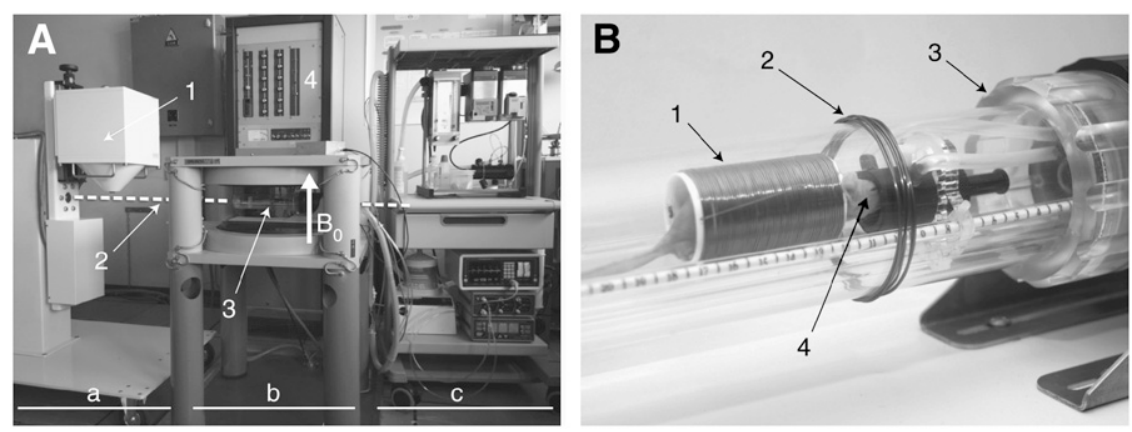

FIGURE 1. (A) General view of hybrid imaging system with pinhole SPECT (a) adjacent on a same-axis configuration to low-field MR imager (b) and close to the anesthesia equipment (c). Imaging cell and animal (3) are translated along the imaging axis (2) from the MRI device to a single-detector pinhole SPECT device (1). Illustration shows orientation of the vertical $B_{0}$ main magnetic field of the MR magnet and corresponding power supply (4). (B) Close-up view of the nonmagnetic imaging cell: solenoidal whole-body RF transmit-and-receive coil (1); pickup coil (2) outside the isolating heated polymethyl methacrylate chamber (3); anesthetic gases are delivered to animal through a dedicated mask (4). 
FIGURE 2. (A) Diagram and coregistrated MR and SPECT images of phantom made of 3 tubes ( $a, b$, and $c$ ) filled with water. One inner tube is filled with $99 \mathrm{mcO}_{4}{ }^{2-}(\mathrm{b})$, whereas the other one is doped with $\mathrm{CuSO}_{4}$ (c). (B) Comparison of ${ }^{99 \mathrm{mTc}}$ and ${ }^{201} \mathrm{TI}$ spectra without (yellow) and with (green) RF coil and imaging cell. A $y$-logarithmic scale was used to enhance the slight differences observed in Compton diffusion for both isotopes. (C) Activity line profile of the single-tube phantom filled with $99 \mathrm{~m} \mathrm{TcO}_{4}{ }^{2-}$ (left) and the same tube rounded by the RF coil inside the imaging cell (right). Low $\gamma$-rays' attenuation of $10 \%$ due to RF coil (a) and imaging cell wall is clearly visible.

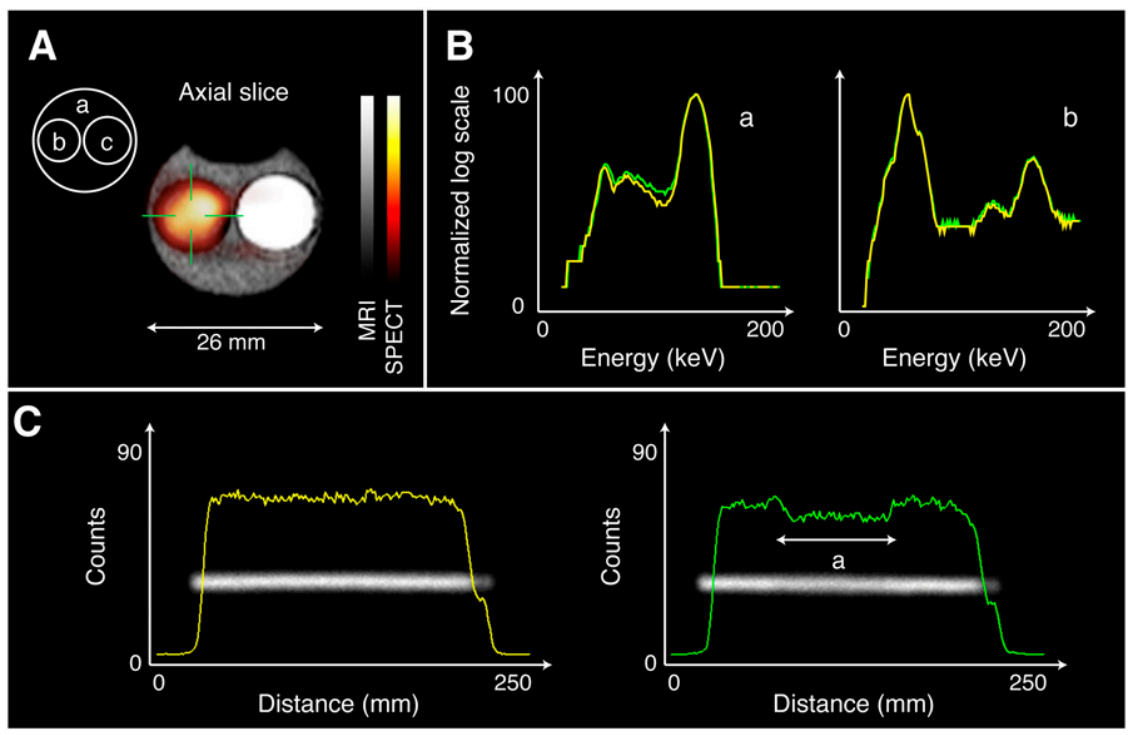

the other. Only one tube of this phantom was filled with $37 \mathrm{MBq}$ of ${ }^{99} \mathrm{mcO}_{4}{ }^{2-}$ for SPECT, whereas the others were filled with water and water doped with $\mathrm{CuSO}_{4}(2 \mathrm{~g} / \mathrm{L})$ for MR T1 contrast enhancement. The T1-weighted imaging sequence defined earlier was used for MRI. For the SPECT study, a $4-\mathrm{cm}$ radius of rotation and a 1.5-mm pinhole diameter were used, and 32 projections of $1 \mathrm{~min}$ were acquired over a circular $180^{\circ}$ arch with a $128 \times 128$ image matrix. Pinhole SPECT images were reconstructed using the EAR method. Then, using AMIDE software (16), rigid coregistration parameters for both datasets were obtained, after identification of common features in each image, by orthogonal $x$-, $y$-, and common $z$-axis translations only and used to correct the centering errors for the in vivo experiments.

\section{Animal Population, MR Contrast Agent, SPECT Radiopharmaceuticals, and Coregistration of Images}

Three adult nude mice, each weighting $25 \pm 1 \mathrm{~g}$ (mean $\pm \mathrm{SD}$ ), were studied using different radiotracers to label different organs and tissues. Pinhole SPECT immediately followed MR acquisition in all experiments. Radiopharmaceuticals with a volume of $0.2 \mathrm{~mL}$ were injected in the tail vein $10 \mathrm{~min}$ before beginning the MR procedure. Corresponding activities were respectively $108 \mathrm{MBq}$ of 99mTc-albumin (Vasculocis; Cis-Bio International) for lung perfusion, $98 \mathrm{MBq}$ of ${ }^{99 \mathrm{~m}} \mathrm{Tc}$-dimercaptosuccinic acid (Renocis; Cis-Bio International) for kidney, and $120 \mathrm{MBq}{ }^{99 \mathrm{~m}} \mathrm{Tc}$-hexamethylpropyleneamine oxime (Ceretec; GE Healthcare) for brain perfusion. Intraperitoneal administration of $1 \mathrm{~mL}$ of $0.1 \mathrm{mmol}$ of gadoteric acid (Dotarem; Guerbet) was used before intravenous injection of radiopharmaceutical for MR tissue contrast enhancement. In all examples, rigid coregistration of images was obtained applying the set of correction values from the 3-tube phantom using the AMIDE software (16). The total acquisition time for SPECT and T1-weighted MR images in mouse lasted $1 \mathrm{~h} 45 \mathrm{~min}$.

\section{RESULTS}

\section{Phantom Studies}

Comparison of total counts in SPECT projection images without and with RF coil and imaging cell show a relative low attenuation of $9.7 \%$ for ${ }^{99 \mathrm{~m}} \mathrm{Tc}$ and $10.5 \%$ for ${ }^{201} \mathrm{Tl}$. The 2-mm wall thickness of the imaging tube cell contributed to $5 \%$ of the total attenuation. In addition, a very slight increase in Compton scattering was observed in both spectra due to the RF coil and wall thickness of the imaging cell tube but without any influence on the counting windows (Fig. 2). Figure 2 also shows the coregistration of SPECT and MR images of the phantom consisting of 3 tubes.

\section{Animal Studies}

Figure 3 shows selected slices of MR T1-weighted wholebody mouse images in which anatomic structures, such as heart cavities, gallbladder, or vertebras, can be easily described. Corrections of off-center misalignment of the imaging cell using the 3-tube phantom data were applied before coregistration of SPECT/MRI mouse images. Anatomic references were then chosen on SPECT and MRI datasets for each experiment, and a mean matching coregistration error of $0.93 \mathrm{~mm}$ was measured using AMIDE software. Figures 4, 5, and 6 illustrate the coregistration of MR and SPECT images in the 3 orthogonal planes for the different radiopharmaceuticals used in this study. The matching of lung, kidney, and brain functional SPECT images on MRI anatomic images demonstrates the practical interest of this dual-modality system.

\section{DISCUSSION}

\section{Spatial Resolution of SPECT and MRI}

Pinhole SPECT is an intrinsic 3D imaging method with isotropic submillimeter-to-millimeter spatial resolution currently achieved in small animals $(1,17-22)$. In our experiments, an isotropic spatial resolution of $1 \mathrm{~mm}$ was obtained by pinhole SPECT with a reconstructed voxel of $0.47 \times 0.47$ $\times 0.47 \mathrm{~mm}^{3}(14,23)$. It is also obvious that isotropic voxel MR images are recommended for optimal coregistration with SPECT. We were able to achieve $0.5 \times 0.5 \times 0.5 \mathrm{~mm}^{3}$ voxels for MRI. They can be compared with $0.3 \times 0.3 \times 0.3 \mathrm{~mm}^{3}$ 


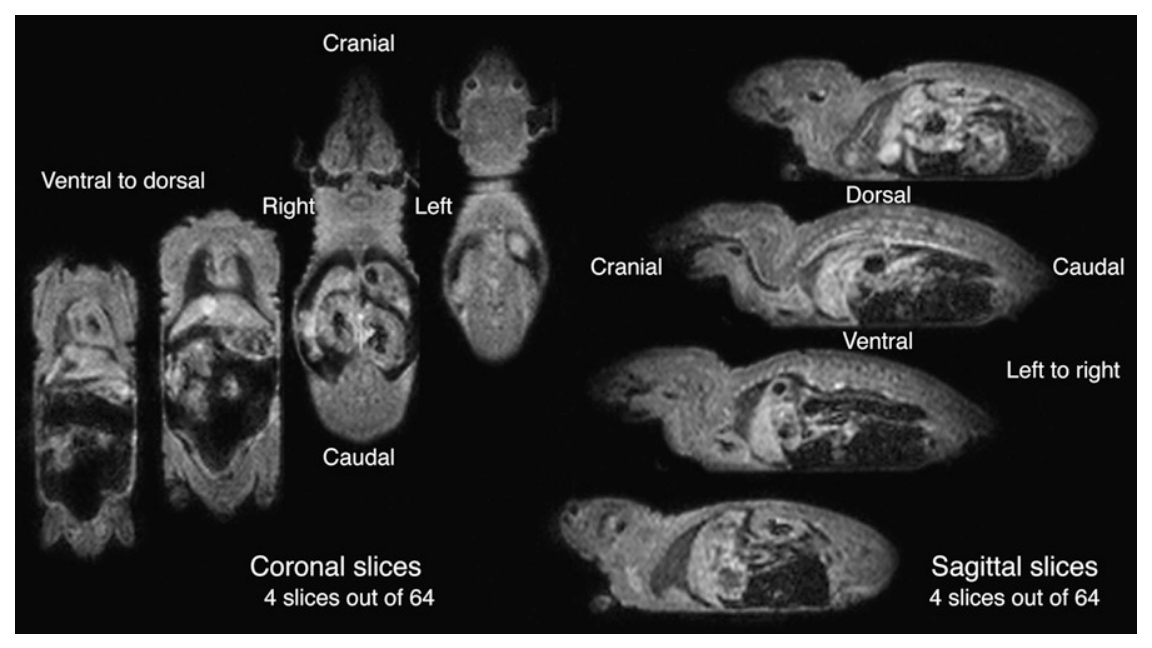

FIGURE 3. Example of selected sagittal and coronal whole-body low-field MRI slices of a nude mouse from the whole set of isotropic $\left(0.5 \times 0.5 \times 0.5 \mathrm{~mm}^{3}\right)$ anatomic T1-weighted images. voxels obtained in mice in separate studies and machines in the only study dealing with MR isotropic voxels, even though this study did not mention the magnetic field strength used (6). Current MRI strategies for small animals are more frequently based on 2-dimensional (2D) multislice acquisition instead of time-consuming isotropic volume imaging sequences. In-plane pixel resolution ranging from 0.15 to $0.25 \mathrm{~mm}$ and slice thickness ranging from 0.7 to $2 \mathrm{~mm}$ were obtained using clinical scanners at $1.5 \mathrm{~T}$ for separate SPECT and MRI rat brain studies $(4,5,7)$. Also, for comparison, a

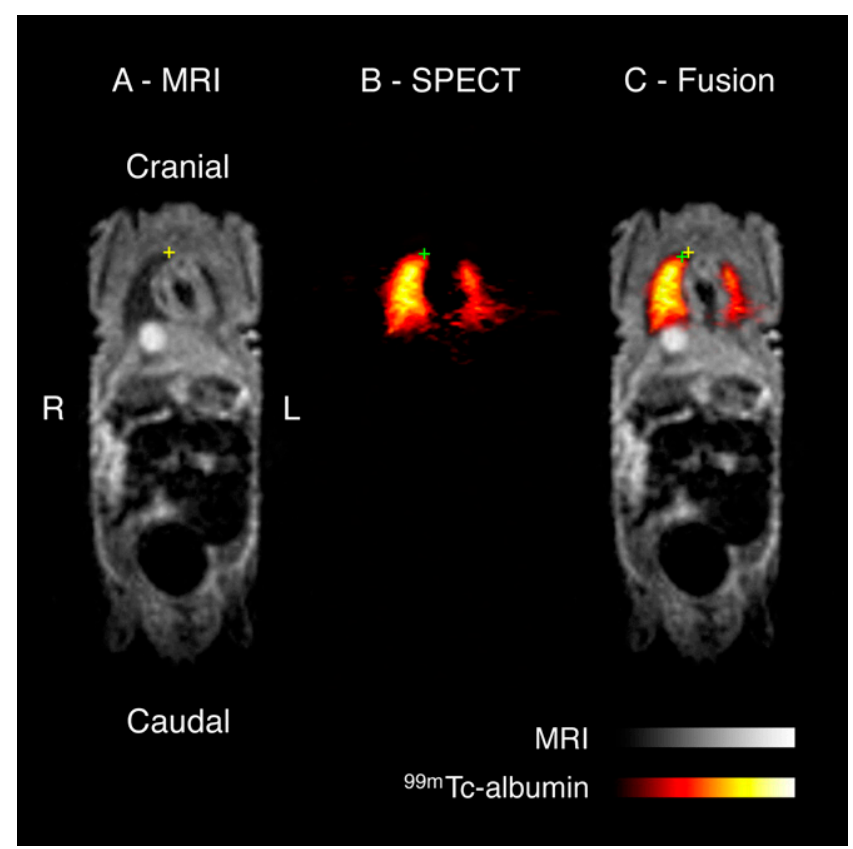

FIGURE 4. (A) T1-weighted low-field MRI coronal slice (in gray) showing lack of signal in lungs and landmark placed manually at apex of right lung. (B) Corresponding SPECT image of lung perfusion (in color) and landmark placed manually at apex of right lung. (C) Fusion of both images where anatomic MRI-based and corresponding functional SPECT landmarks are shown on this particular slice assessment of coregistration error calculated as mean distance between 4 coregistered landmarks per complete image dataset.
$0.8 \times 0.8 \times 0.8 \mathrm{~mm}^{3}$ voxel has been used for a whole-body mouse MR acquisition in separate PET and MRI studies using a 4.7-T magnetic field (24). However, SPECT/MRI fusion studies based on nonisotropic voxels for both modalities lack precision for image coregistration and fusion because the MRI slice thickness differs from the pixel size, even if the MR in-plane pixel resolution is improved. Our results show that the voxel size achieved at $0.1 \mathrm{~T}$ is adapted for an easy routine coregistration for SPECT functional images with anatomic MR references. As physiologic movements of

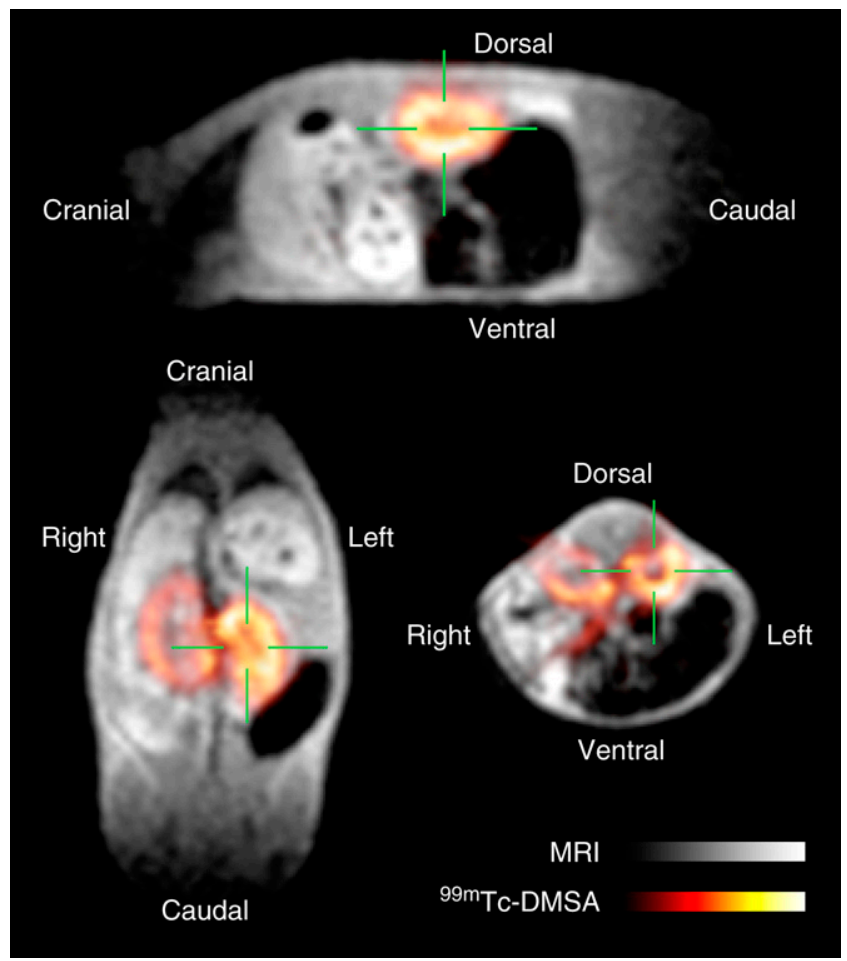

FIGURE 5. Selected coregistered orthogonal slices of pinhole SPECT ( ${ }^{99 m}$ Tc-dimercaptosuccinic acid [ ${ }^{99 m}$ Tc-DMSA] kidney uptake in color) and low-field MRI (soft-tissue anatomic information in gray) in nude mouse. 


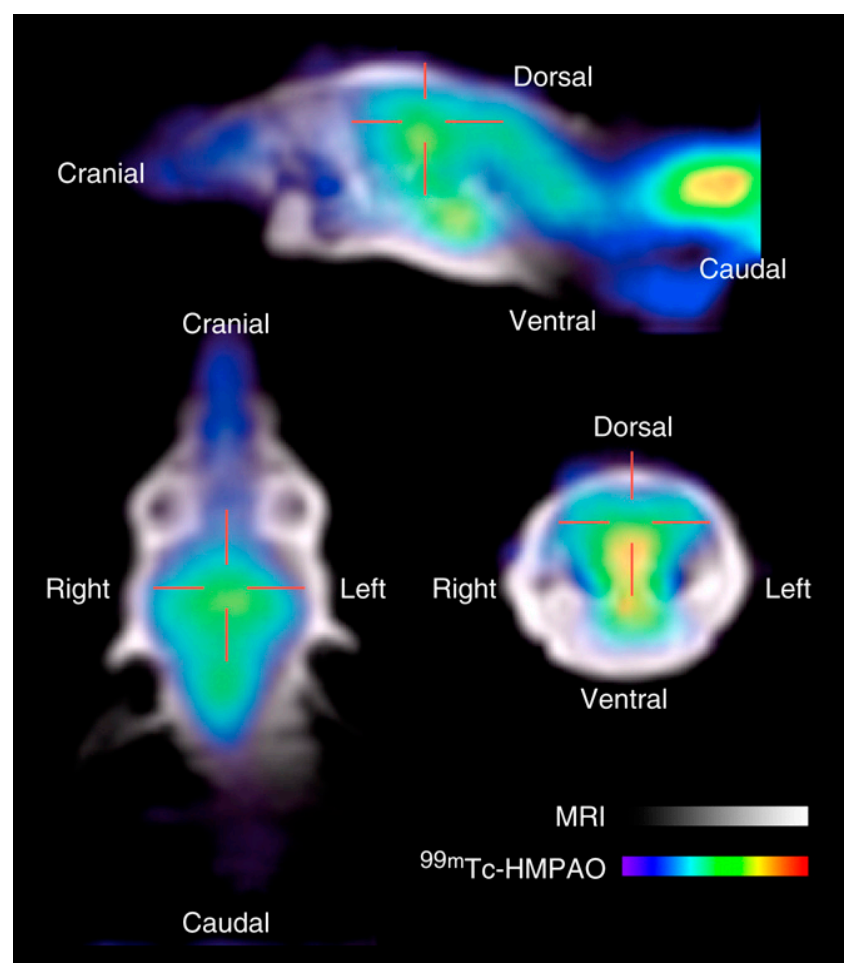

FIGURE 6. Selected coregistered orthogonal slices of pinhole SPECT (brain perfusion in color) and low-field MRI (soft-tissue anatomic information in gray) in nude mouse. HMPAO = hexamethylpropyleneamine oxime.

the animal and tissues due to circulation, respiration, and fluid flow cannot be systematically controlled, it also seems unrealistic to aim for MR spatial in-plane resolution below $0.2 \times 0.2 \mathrm{~mm}^{2}(25,26)$. Compared with high and very high magnetic fields, dealing with the relative voxel sizes achieved by each of the combined technologies also does not make sense to merge SPECT data, whose spatial resolutions are on the order of $1 \mathrm{~mm}^{3}$, with MR spatial resolutions of $0.001 \mathrm{~mm}^{3}$. Of course, the major, but almost only, disadvantage of a low magnetic field compared with a high field (proton spectroscopic information notwithstanding) is the reduction in the signal-to-noise $(\mathrm{S} / \mathrm{N})$ ratio. However, improvements with regard to the $\mathrm{S} / \mathrm{N}$ ratio and resolution at low magnetic field are possible by increasing the gradient strength, which increases both voxel resolution and signal because it shortens the echo time. The use of superconducting or copper-cooled RF coils also allows an increase in the $\mathrm{S} / \mathrm{N}$ ratio but remains questionable because of the necessity of heating the imaging cell at the same time $(27,28)$.

\section{Total Acquisition Time for SPECT/MRI}

In our experience, the total acquisition time of the complete set of SPECT/MR images was $<2 \mathrm{~h}$. As we used the technical imaging cell for both modalities, the manual transfer of the animal from SPECT to MRI was effective in about $1 \mathrm{~min}$, but this translation could also be done automatically as SPECT and MRI components are adjacent to one another on the same imaging axis. The acquisition time of SPECT images alone could also be optimized in view of recent technical improvements—such as multiple heads' $\mathrm{NaI}$ crystal detectors, multiple pinholes, or multiple semiconductor detectors-which can increase the sensitivity of $\gamma$-photon detection (18-22). In our experience, total acquisition times with low-field MRI were identical to the $43 \mathrm{~min}$ in the experience of isotropic voxel high-field MRI used for PET/MRI coregistration images in mice (24). Obviously, multislice 2D MR acquisitions are faster (about $15 \mathrm{~min}$, for instance, at $1.5 \mathrm{~T}(4,5))$ but, as mentioned earlier, are not well adapted for adequate coregistration of images because the MRI voxel needs to be resized to match the isotropic SPECT voxel size. Moreover, 3D MRI sequences had a better $\mathrm{S} / \mathrm{N}$ ratio relative to the acquisition time than corresponding $2 \mathrm{D}$ multislice sequences, which is an advantage when using a low magnetic field strength but at the expense of longer acquisition times (29).

\section{Imaging Cell for Both Online Sequential Modalities}

Keeping the animal motionless was achieved by the use of the imaging cell, allowing easy, safe, and rapid transport of the anesthetized animal from one imager to anothertherefore, minimizing the delay between both sets of image acquisitions. As the animal remains anesthetized in the imaging cell from the beginning of the sequential imaging modalities, the lack of fiducial markers inside the imaging cell was not critical for coregistration of SPECT/MR images in our experiments. It is true that we found a mean coregistration error corresponding to the value of the spatial resolution of the pinhole SPECT, which is the less-resolved modality. However, use of a nonrigid spatial transformation matrix based on grid phantoms and fiducial markers could be easily mounted on the imaging cell to facilitate improvement in the accuracy of images' coregistration as done by Chow et al. (30). Finally, the use of the imaging cell enables registration of both sets of images during the same anesthesia session, thus avoiding various biologic responses due to different doses and types of anesthesia as could be the case in completely separate acquisitions (3).

\section{Small-Animal Control and Survey During SPECT/MRI Dual Imaging}

A specific advantage of the combined pinhole SPECT and dedicated low-field MR imager used in this study is that the open magnet enables, as for the pinhole SPECT, an easy placement and a continuous visual monitoring of the small animal during the entire imaging procedure.

\section{Sequential Versus Simultaneous SPECT/MRI}

It is recognized that within the general field of molecular imaging there is a need for methods of coregistering MRI with nuclear imaging modalities, but use of high magnetic fields for simultaneous imaging (MRI and SPECT) places important constraints on many engineering and cost aspects of a system design as opposed to the dual device used in this study $(3,10,31)$. The low-field MRI system and the MR-compatible animal holder we propose also had the 
advantages of being well adapted to an integrated coaxial rail-based dual device or a docking and click-over modular approach for combining sequential small-animal SPECT and MRI (32). Recent results have shown that semiconductor detectors (cadmium-zinc-telluride [CZT]) that can be used for small-animal SPECT were also insensitive to magnetic fields up to $7 \mathrm{~T}$, leading to the development of a prototype limited to 24 unique angular samples but opening the way for simultaneous SPECT/MRI systems $(3,10)$. Smallanimal truly simultaneous versus sequential SPECT/MRI applications are actually difficult to define. This is mainly due to the constant technologic evolution for both pinhole SPECT and MR instrumentation to increase their sensitivities while minimizing the duration of image acquisition.

\section{CONCLUSION}

In this article we have shown that a small-animal SPECT/ MRI dual hybrid device combining a pinhole SPECT imager adjacent to a small low-field MR imager is a low-cost potential solution for anatomic and functional combination that can be useful in molecular preclinical imaging.

\section{ACKNOWLEDGMENTS}

This research was performed during the tenure of a doctoral fellowship from GE Healthcare and Région Alsace for E. Breton. The authors thank Minerve and Cis-Bio International for support and Jacqueline Kintz for her help.

\section{REFERENCES}

1. Meikle S, Kench P, Kassiou M, Banati R. Small animal SPECT in the matrix of molecular imaging technologies. Phys Med Biol. 2005;50:R45-R61.

2. Marzola P, Osculati F, Sbarbati A. High field MRI in preclinical research. Eur J Radiol. 2003;48:165-170.

3. Wagenaar D, Kapusta M, Li J, Patt B. Rationale for the combination of nuclear medicine with magnetic resonance for pre clinical imaging. Technol Cancer Res Treat. 2006;5:343-350.

4. Scherfler C, Donnemiler E, Schocke M, et al. Evaluation of striatal dopamine transporter function in rats by in vivo beta-CIT pinhole SPECT. Neuroimage. 2002;17:128-141.

5. Scherfler C, Scholtz S, Donnemiler E, et al. Evaluation of ${ }^{123}$ I-IBZM pinhole SPECT for the detection of striatal dopamine D2 receptor availability in rats. Neuroimage. 2005;24:822-831.

6. Schurrat T, Alfke H, Béhé M, et al. Molecular gastrin receptor localization in mice using high resolution SPET-MRI image fusion. Eur J Nucl Med Mol Imaging. 2003;30:800.

7. Booij J, De Bruin K, De Win M, Lavini C, Den Heeten G, Habraken J. Imaging of striatal dopamine transporters in rat brain with single pinhole SPECT and coaligned MRI is highly reproducible. Nucl Med Biol. 2003;30:643-649.
8. Hansen G, Crooks L, Davis P, et al. In vivo imaging of the rat anatomy with nuclear magnetic resonance. Radiology. 1980;136:695-700.

9. Benveniste H, Blackband S. MR microscopy and high resolution small animal MRI: applications in neuroscience research. Prog Neurobiol. 2002;67:393-420.

10. Wagenaar D, Nalcioglu O, Muftuler L, et al. A multi-ring small animal CZT system for simultaneous SPECT/MRI imaging [abstract]. J Nucl Med. 2007; 48(suppl):89P.

11. Marsden P, Strul D, Keevil S, Williams M, Cash D. Simultaneous PET and NMR. Br J Radiol. 2002;75:S53-S59.

12. Catana C, Wu Y, Judenhofer M, Qi J, Pichler B, Cherry S. Simultaneous acquisition of multislice PET and MR images: initial results with a MRcompatible PET scanner. J Nucl Med. 2006;47:1968-1976.

13. Breton E, Choquet P, Goetz C, et al. Dual SPECT/MR imaging in small animal. Nucl Instrum Methods Phys Res A. 2007;571:446-448.

14. Israel-Jost V, Choquet P, Salmon S, Blondet C, Sonnendrucker E, Constantinesco A. Pinhole SPECT imaging: compact projection/backprojection operator for efficient algebraic reconstruction. IEEE Trans Med Imaging. 2006;25:158-167.

15. Arbogast-Ravier S, Xu F, Choquet P, Brunot B, Constantinesco A. Dedicated low field MRI: a promising low-cost technique. Med Biol Eng Comput. 1995;33:735739.

16. Loening A, Gambhir S. AMIDE: a free software tool for multimodality medical image analysis. Mol Imaging. 2003;2:131-137.

17. Green M, Seidel J, Vaquero J, Jagoda E, Lee I, Eckelman W. High resolution PET, SPECT and projection imaging in small animals. Comput Med Imaging Graph. 2001;25:79-86.

18. Kim H, Furenlid L, Crawford M, et al. SemiSPECT: a small animal single photon emission computed tomography (SPECT) imager based on eight cadmium zinc telluride (CZT) detector arrays. Med Phys. 2006;33:465-474.

19. Kubo N, Zhao S, Fujiki Y, et al. Evaluating performance of a pixel array semiconductor SPECT system for small animal imaging. Ann Nucl Med. 2005;19: 633-639.

20. Schramm N, Ebel G, Engeland U, Schurrat T, Behe M, Behr T. High resolution SPECT using multipinhole collimation. IEEE Trans Nucl Sci. 2003;50:315-320.

21. Beekman FJ, van der Have F, Vastenhouw B, et al. U-SPECT-I: a novel system for submillimeter-resolution tomography with radiolabeled molecules in mice. J Nucl Med. 2005;46:1194-1200.

22. Walrand S, Jamar F, De Jong M, Pauwels S. Evaluation of novel whole body high resolution rodent SPECT (linoview) based on direct acquisition of linogram projections. J Nucl Med. 2005;46:1872-1880.

23. Constantinesco A, Choquet P, Monassier L, Israel-Jost V, Mertz L. Assessment of left ventricular perfusion, volumes, and motion in mice using pinhole gated SPECT. J Nucl Med. 2005;46:1005-1011.

24. Rowland D, Garbow J, Laforest R, Snyder A. Registration of ${ }^{18}$ F-FDG microPET and small animal MRI. Nucl Med Biol. 2005;32:567-572.

25. Acton PD. Animal imaging equipment: recent advances. J Nucl Med. 2006; 47(12):52N-55N.

26. Mai W, Badea C, Wheeler C, Hedlund L, Johnson G. Effects of breathing and cardiac motion on spatial resolution in the microscopic imaging of rodents. Magn Reson Med. 2005;53:858-865.

27. Bourne L. Estimated SNR gains at $0.2 \mathrm{~T}$ from superconducting coils. Proceedings of the International Society for Magnetic Resonance in Medicine: 4th Scientific Meeting; April 27-May 3, 1996; New York, NY. Abstract 1449.

28. Wright A, Song H, Wehrli F. In vivo MR micro imaging with conventional radiofrequency coils cooled to $77^{\circ} \mathrm{K}$. Magn Reson Med. 2000;43:163-169.

29. Johnson G, Thomson M, Drayer P. Three-dimensional MRI microscopy of the normal rat brain. Magn Reson Med. 1987;4:351-365.

30. Chow P, Stout B, Komisopoulou E, Chatziioannou A. A method of image registration for small animal, multi-modality imaging. Phys Med Biol. 2006;51:379-390.

31. Gore J. Role of MR molecular imaging. J Nucl Med. 2006;47(12):44N-45N.

32. Beekman F, Hutton F. Multi-modality imaging on track. Eur J Nucl Med Mol Imaging. 2007;34:1410-1414. 